\title{
The role of ATP-sensitive potassium channels on DMT1-mediated ferrous iron uptake in SK-N-SH cells
}

\author{
Xixun Du, Huamin Xu, Ning Song, Hong Jiang, Junxia Xie* \\ From 2011 International Conference on Molecular Neurodegeneration \\ Shanghai, China. 22-24 September 2011
}

\section{Background}

Elevated iron accumulation has been reported in the substantia nigra (SN) in Parkinson's disease (PD). Our previous study observed that increased levels of the iron importer divalent metal transporter 1 (DMT1) were involved in this nigral iron accumulation and dopaminergic neurons loss in PD. The iron transport function of DMT1 is also related to the membrane potential level, which increases with hyperpolarization of the cell membrane. Activation of ATP-sensitive potassium $\left(\mathrm{K}_{\mathrm{ATP}}\right)$ channels, which could induce hyperpolarization of nigral dopaminergic neurons, is reported to be involved in the selective loss of these neurons in PD. The present study is to investigate whether activation of $\mathrm{K}_{\mathrm{ATP}}$ channels could change the iron uptake function of DMT1.

\section{Methods}

DiBAC4(3) and calcein were used to detect membrane potential and ferrous iron influx. Intracellular iron concentration was measured using an inductively coupled plasma (ICP-2) detector. Mitochondrial transmembrane potential $(\triangle \Psi \mathrm{m})$ and reactive oxygen species (ROS) were measured by flow cytometry using rhodamine 123 and $\mathrm{H}_{2}$ DCF-DA.

\section{Results}

(1) When treated with diazoxide, a novel $\mathrm{K}_{\mathrm{ATP}}$ channel opener, the membrane potential of SK-N-SH cells showed hyperpolarization. (2) The influx of ferrous iron and the intracellular iron levels were observed dramatically increased when the cells were co-incubated with diazoxide and ferrous iron, resulting in a decreased $\Delta \Psi \mathrm{m}$ and an elevated level of ROS production. (3) When treated with diazoxide, cells with DMT1 knockdown showed decreased ferrous iron influx compared with the vector control. (4) When treated with diazoxide, cells overexpressed of SUR1 and Kir6.2 showed increased ferrous iron influx compared with the vector control, which induced a decrease in $\Delta \Psi \mathrm{m}$ and an increase in ROS production.

\section{Conclusion}

These results suggest that the activation of the $\mathrm{K}_{\mathrm{ATP}}$ channels could enhance DMT1-mediated ferrous iron uptake, leading to increase intracellular oxidative stress.

\section{Acknowledgements}

This work was supported by grants from the National Program of Basic Research sponsored by the Ministry of Science and Technology of China (2011CB504100), the National Foundation of Natural Science of China (30930036) and the Natural Science Fund of Shandong Province for Distinguished Young Scholars (JQ200807).

Published: 7 February 2012

doi:10.1186/1750-1326-7-S1-S6

Cite this article as: Du et al:: The role of ATP-sensitive potassium channels on DMT1-mediated ferrous iron uptake in SK-N-SH cells. Molecular Neurodegeneration 2012 7(Suppl 1):S6.

\footnotetext{
* Correspondence: jxiaxie@public.qd.sd.cn

Department of Physiology, Shandong Provincial Key Laboratory of Pathogenesis and Prevention of Neurological Disorders and State Key Disciplines: Physiology, Medical College of Qingdao University, Qingdao 266071, China
}

(c) 2012 Du et al; licensee BioMed Central Ltd. This is an Open Access article distributed under the terms of the Creative Commons 